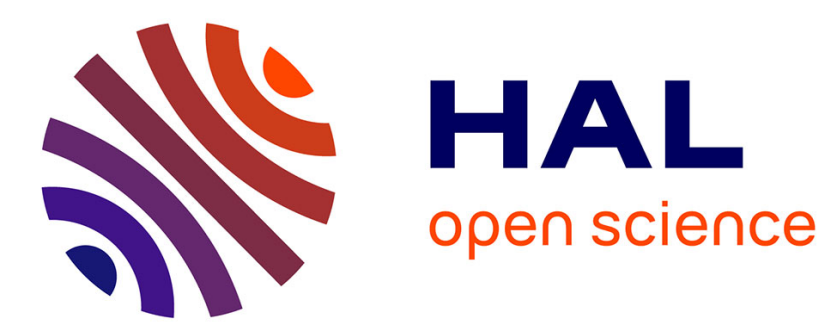

\title{
Upscaling of spectral induced polarization response using random tube networks
}

Alexis Maineult, André Revil, Christian Camerlynck, Nicolas Florsch, Konstantin Titov

\section{- To cite this version:}

Alexis Maineult, André Revil, Christian Camerlynck, Nicolas Florsch, Konstantin Titov. Upscaling of spectral induced polarization response using random tube networks. Geophysical Journal International, 2017, 209 (2), pp.948-960. 10.1093/gji/ggx066 . hal-01494869

\section{HAL Id: hal-01494869 \\ https://hal.sorbonne-universite.fr/hal-01494869}

Submitted on 24 Mar 2017

HAL is a multi-disciplinary open access archive for the deposit and dissemination of scientific research documents, whether they are published or not. The documents may come from teaching and research institutions in France or abroad, or from public or private research centers.
L'archive ouverte pluridisciplinaire HAL, est destinée au dépôt et à la diffusion de documents scientifiques de niveau recherche, publiés ou non, émanant des établissements d'enseignement et de recherche français ou étrangers, des laboratoires publics ou privés. 


\title{
Upscaling of spectral induced polarization response using random tube networks
}

\author{
Alexis Maineult, ${ }^{1}$ André Revil, ${ }^{2}$ Christian Camerlynck, ${ }^{1}$ Nicolas Florsch ${ }^{1,3}$ \\ and Konstantin Titov ${ }^{4}$ \\ ${ }^{1}$ Sorbonne Universités, UPMC Univ Paris 06, CNRS, EPHE, UMR 7619 Metis, 4 place Jussieu, F-75005 Paris, France. E-mail: alexis.maineult@upmc.fr \\ ${ }^{2}$ ISTerre, CNRS, UMR CNRS 5275, Université de Savoie Mont-Blanc, F-73370 Le Bourget du Lac, France \\ ${ }^{3}$ Sorbonne Universités, UPMC Univ Paris 06, IRD, UMI 209 UMMISCO, 32 Av. Henry Varagnat, F-93140 Bondy, France \\ ${ }^{4}$ Institute of Earth Sciences, St. Petersburg State University, 7-9 Universitetskaya naberezhnaya, 199034 St. Petersburg, Russia
}

Accepted 2017 February 16. Received 2017 February 15; in original form 2016 July 13

\begin{abstract}
SUMMAR Y
In order to upscale the induced polarization (IP) response of porous media, from the pore scale to the sample scale, we implement a procedure to compute the macroscopic complex resistivity response of random tube networks. A network is made of a 2-D square-meshed grid of connected tubes, which obey to a given tube radius distribution. In a simplified approach, the electrical impedance of each tube follows a local Pelton resistivity model, with identical resistivity, chargeability and Cole-Cole exponent values for all the tubes-only the time constant varies, as it depends on the radius of each tube and on a diffusion coefficient also identical for all the tubes. By solving the conservation law for the electrical charge, the macroscopic IP response of the network is obtained. We fit successfully the macroscopic complex resistivity also by a Pelton resistivity model. Simulations on uncorrelated and correlated networks, for which the tube radius distribution is so that the decimal logarithm of the radius is normally distributed, evidence that the local and macroscopic model parameters are the same, except the Cole-Cole exponent: its macroscopic value diminishes with increasing heterogeneity (i.e. with increasing standard deviation of the radius distribution), compared to its local value. The methodology is also applied to six siliciclastic rock samples, for which the pore radius distributions from mercury porosimetry are available. These samples exhibit the same behaviour as synthetic media, that is, the macroscopic Cole-Cole exponent is always lower than the local one. As a conclusion, the pore network method seems to be a promising tool for studying the upscaling of the IP response of porous media.
\end{abstract}

Key words: Electrical properties; Hydrogeophysics; Numerical modelling.

\section{INTRODUCTION}

Induced polarization (IP) method is now commonly used in a broad range of geophysical applications, for instance ore or hydrocarbon prospection (e.g. Veeken et al. 2009), location and delineation of contaminant plumes (e.g. Mewafy et al. 2013; Johansson et al. 2015; Ntarlagiannis et al. 2016), monitoring of biodegradation processes in contaminated area (e.g. Flores Orozco et al. 2011; Abdel Aal et al. 2014) and even hydrological characterization of the subsurface (e.g. Attwa \& Günther 2013).

A recently developed version of the method - the spectral induced polarization (SIP) method — consists in injecting a sinusoidal current $I$ in a rock sample (in laboratory conditions) or in the subsurface (in field conditions) by means of two current injection electrodes, and in measuring the resulting voltage difference $\Delta V$ by means of at least two measurement electrodes. Doing so for different frequencies, one obtains the spectrum of the complex resistivity of the medium $\rho^{*}$. In the complex domain,

$\rho^{*}(\omega)=K \frac{\Delta V^{*}(\omega)}{I^{*}(\omega)}=K \frac{\left|\Delta V^{*}(\omega)\right|}{\left|I^{*}(\omega)\right|} e^{-i \varphi(\omega)}=\rho(\omega) e^{-i \varphi(\omega)}$,

where superscript $*$ refers to complex quantities, $K$ is a geometrical constant linked among others to the location of the electrodes, $\omega$ is the pulsation frequency, $\rho$ is the resistivity amplitude, $\varphi$ is the phase and $i=(-1)^{\frac{1}{2}}$ is the pure imaginary number. Amplitude and phase spectra are related to the ability of the material to store electrical charges reversibly.

Understanding large-scale measurements (field and rock samples) requires the knowledge of the polarization processes that occur at the pore scale, together with the development of appropriate 
upscaling procedures. At frequencies lower than $100 \mathrm{~Hz}$, and in absence of metallic particles (which act as strong capacitors when submitted to a low-frequency current, e.g. Wong 1979; Misra et al. 2016a,b), the main polarization mechanisms are (1) the polarization of the Stern layer at the interface between the mineral grains and the saturating fluid (e.g. Rosen \& Saville 1991; Revil 2012, 2013), and (2) the membrane polarization, which is controlled by the differential diffusion of cations and anions through small pores (e.g. Marshall \& Madden 1959; Bücker \& Hördt 2013a). Many models have been proposed to formalize these two phenomena at the pore scale (e.g. Marshall \& Madden 1959; Titov et al. 2002; Revil \& Florsch 2010; Bücker \& Hördt 2013a,b). In addition, a broad variety of semi-empirical models have been published to reproduce the observed IP response of rock samples (e.g. Pelton et al. 1978; Dias 2000; Weller et al. 2013; Weller \& Slater 2015) in the quasi-static approximation (below $1000 \mathrm{~Hz}$ ). Upscaling methods used to link the models at the pore scale and the models at the sample scale are usually based on effective medium approximation (e.g. Dukhin \& Shilov 2002) - for instance, the differential effective medium approach for suspensions of grains in a background electrolyte. For heterogeneous media, the issue of upscaling is still open.

In this paper, we use the method of random pore networks to address this question of upscaling. In other words, based on a known complex impedance function at the pore scale, we model the complex impedance (or complex resistivity) function of the macroscopic assemblage of pores. Note that the same procedure could be applied to a suspension of spheres or spheroidal grains (with their electrical double layer) in a background electrolyte as long as the complex impedance function of the basic element is known. Pore networks have been extensively used to study the permeability and the formation factor of rocks (e.g. Kirkpatrick 1973; Koplik 1981; David et al. 1990; David 1993; Bernabé et al. 2003), as well as the relationship between electrical conductivity and permeability (Bernabé \& Revil 1995), their transport properties (e.g. Bruderer \& Bernabé 2001; Bernabé et al. 2016) and even their self-potential response (Bernabé 1998; Jackson 2010). Briefly, the method consists in designing 2-D or 3-D networks of connected tubes, for a given connectivity (linked to the topology, i.e. to the type of mesh: triangular, square, hexagonal and so on), and to attribute to the tubes a given distribution of radii (and/or lengths), similar to the pore radius distribution that can be observed in rocks. The two end face of the network are then submitted to appropriate boundaries conditions, and the conservation law (for hydraulic flow or electrical current) is applied to each node to which tubes are connected. This yields to a linear system of field equations enabling the potential (hydraulic or electrical) to be solved, from which the hydraulic flow or electrical current in the tubes are inferred, and therefore the macroscopic fluxes at the end faces of the macroscopic body. From these fluxes, the parameter of interest, such as the permeability or the formation factor, can be deduced like in a real laboratory experiment. Even though using the network method obviously assumes strong simplifications, in particular concerning the topology of the simulated medium, it has some advantages. The method is very simple. It presents the possibility to carry out a huge number of simulations on different network realizations for a given law of radius distribution, and thus to obtain statistics. Finally, the influence of the topology and the shape of the radius distribution can be extensively studied (e.g. Bernabé \& Bruderer 1998; Bernabé et al. 2003), which is almost impossible with real rocks. In this way, these conceptual tools permit general laws to be evidenced (e.g. Bernabé et al. 2010, 2011).

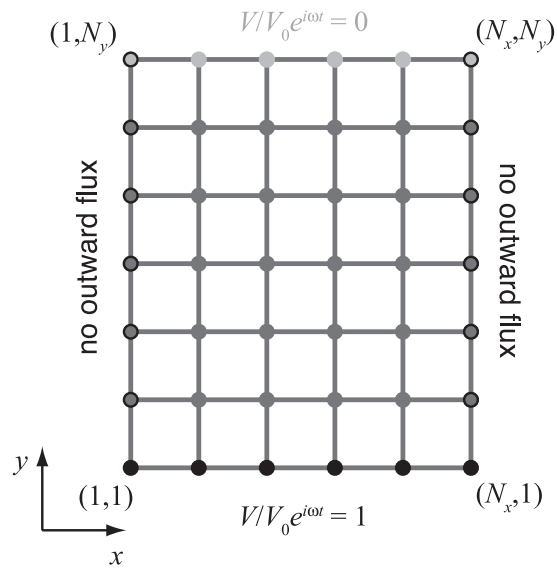

Figure 1. 2-D square network of connected tubes. Each tube can be used to represent a pore. The electrical potential is imposed at the nodes in entry $(y=1)$ and exit $\left(y=N_{y}\right)$. There are no outflowing currents on the lateral faces $\left(x=1\right.$ and $x=N_{x}$ ). The radii of the tubes follow a given distribution, which can be for instance derived from mercury porosimetry or nuclear magnetic resonance.

\section{METHODOLOGY}

\subsection{Tube network}

We consider a simulated porous material with a pore network composed of a set of connected capillaries. More precisely, we consider 2-D square networks (Fig. 1), made of a set of $N_{x} \cdot N_{y}$ nodes $\left(N_{x}\right.$ in the $x$-direction and $N_{y}$ in the $y$-direction). These nodes are connected to each other by $2 N_{x} \cdot N_{y}-N_{x}-N_{y}$ tubes representing the pores. Note that we use 2-D networks and not 3-D ones to allow larger network size, but the methodology is extendable to 3-D. The network is submitted to given boundary conditions such as insulating boundaries on some sides and with an imposed voltage on others like in a real experiment. The radii of the tubes obey to a pore size distribution and they are randomly chosen on this (normalized) probability distribution. An example of a non-correlated random network is shown in Fig. 2, together with the associated 'experimental' radius distribution (Fig. 2a). In laboratory experiments, these experimental radius distributions can be determined either from mercury porosimetry (using the capillary pressure curve, e.g. Ritter \& Drake 1945) or from nuclear magnetic resonance measurements using the distribution of the relaxation times (e.g. Gallegos et al. 1987).

The principle of a simulation consists in solving the (complex valued) electrical potential at the nodes, to deduce the electrical current through the tubes, from which the macroscopic IP response of the network can be inferred. In our case, the tubes are characterized by a complex impedance function, which is supposed to be known. The central questions that we will address are: (1) how to upscale the problem to get the complex impedance of the network and (2) can we describe the macroscopic impedance function from the local impedance function and from the properties of the network such as the pore size distribution.

\subsection{System for the complex electrical potential}

Kirchhoff law (Kirchhoff 1845) for the complex electrical current, applied at each node $(x, y)$ inside the internal domain of the network 


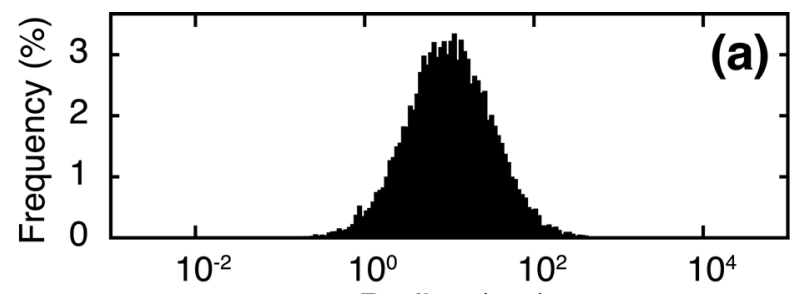

(b)
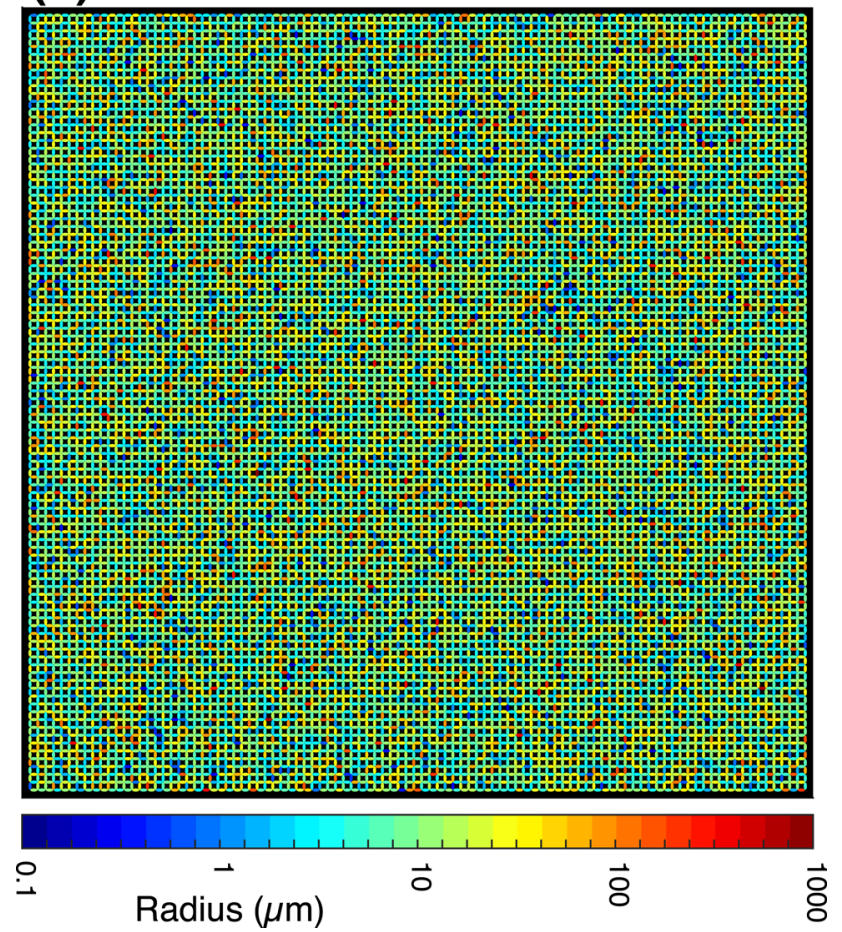

Figure 2. Example of random uncorrelated media. 'Experimental' distribution (a) of the tube radii (the decimal logarithm of the pore tube radius distribution is normally distributed, with a mean radius of $10 \mu \mathrm{m}$ and a standard deviation of 0.5$)$ associated with the network $(100 \times 100$ nodes, 19800 tubes) shown in (b).

(Fig. 1), writes:

$$
\begin{aligned}
& \forall(x, y) \in\left[2, \ldots, N_{x}-1\right] \times\left[2, \ldots, N_{y}-1\right] \\
& \quad J_{(x, y-1) \rightarrow(x, y)}^{*}+J_{(x-1, y) \rightarrow(x, y)}^{*}+J_{(x+1, y) \rightarrow(x, y)}^{*}+J_{(x, y+1) \rightarrow(x, y)}^{*}=0
\end{aligned}
$$

where $J_{p \rightarrow q}^{*}$ is the electrical current inside the tube relying node $p$ to node $q$. The local constitutive equation (Ohm's law) is

$J_{p \rightarrow q}^{*}=\sigma_{p \rightarrow q}^{*}\left(V_{p}^{*}-V_{q}^{*}\right)$,

where $\sigma_{p \rightarrow q}^{*}$ is the complex conductivity of the tube (including not only the fluid, but also the effect of the embedding mineral) and $V_{p}^{*}\left(\right.$ respectively $V_{q}^{*}$ ) is the complex electrical potential at node $p$ (respectively $q$ ). Considering eq. (3), eq. (2) becomes:

$$
\begin{gathered}
\sigma_{(x, y-1) \rightarrow(x, y)}^{*} V_{x, y-1}^{*}+\sigma_{(x-1, y) \rightarrow(x, y)}^{*} V_{x-1, y}^{*}+\Sigma_{x, y}^{*} V_{x, y}^{*} \\
+\sigma_{(x+1, y) \rightarrow(x, y)}^{*} V_{x+1, y}^{*}+\sigma_{(x, y+1) \rightarrow(x, y)}^{*} V_{x, y+1}^{*}=0,
\end{gathered}
$$

where

$$
\begin{aligned}
\Sigma_{x, y}^{*}= & -\left(\sigma_{(x, y-1) \rightarrow(x, y)}^{*}+\sigma_{(x-1, y) \rightarrow(x, y)}^{*}+\sigma_{(x+1, y) \rightarrow(x, y)}^{*}\right. \\
& \left.+\sigma_{(x, y+1) \rightarrow(x, y)}^{*}\right) .
\end{aligned}
$$

The boundary conditions applied at the entry (i.e. the nodes for which $y=1$ ) and at the exit (i.e. the nodes for which $y=N_{y}$ ) are (see Fig. 1):

$\forall x \in\left[1, \ldots, N_{x}\right] \quad V_{x, 1}^{*}=V_{0} e^{i \omega t}$

$\forall x \in\left[1, \ldots, N_{x}\right] \quad V_{x, N_{y}}^{*}=0$

where $V_{0}$ is the amplitude (real number), $\omega$ is the pulsation frequency and $t$ stands for time. On the lateral faces, there are no outward fluxes:

$\forall y \in\left[2, \ldots, N_{y}-1\right]\left\{\begin{array}{c}J_{(1, y-1) \rightarrow(1, y)}^{*}+J_{(2, y) \rightarrow(1, y)}^{*} \\ +J_{(1, y+1) \rightarrow(1, y)}^{*}=0 \\ J_{\left(N_{x}, y-1\right) \rightarrow\left(N_{x}, y\right)}^{*}+J_{\left(N_{x}-1, y\right) \rightarrow\left(N_{x}, y\right)}^{*} \\ +J_{\left(N_{x}, y+1\right) \rightarrow\left(N_{x}, y\right)}^{*}=0\end{array}\right.$,

that is:

$$
\begin{aligned}
& \sigma_{(1, y-1) \rightarrow(1, y)}^{*} V_{1, y-1}^{*}+\Sigma_{1, y}^{*} V_{1, y}^{*}+\sigma_{(2, y) \rightarrow(1, y)}^{*} V_{2, y}^{*} \\
& \quad+\sigma_{(1, y+1) \rightarrow(1, y)}^{*} V_{1, y+1}^{*}=0,
\end{aligned}
$$

with

$\Sigma_{1, y}^{*}=-\left(\sigma_{(1, y-1) \rightarrow(1, y)}^{*}+\sigma_{(2, y) \rightarrow(1, y)}^{*}+\sigma_{(1, y+1) \rightarrow(1, y)}^{*}\right)$,

and

$$
\begin{aligned}
& \sigma_{\left(N_{x}, y-1\right) \rightarrow\left(N_{x}, y\right)}^{*} V_{N_{x}, y-1}^{*}+\sigma_{\left(N_{x}-1, y\right) \rightarrow\left(N_{x}, y\right)}^{*} V_{N_{x}-1, y}^{*}+\Sigma_{N_{x}, y}^{*} V_{N_{x}, y}^{*} \\
& \quad+\sigma_{\left(N_{x}, y+1\right) \rightarrow\left(N_{x}, y\right)}^{*} V_{N_{x}, y+1}^{*}=0,
\end{aligned}
$$

with

$\Sigma_{N_{x}, y}^{*}=-\left(\sigma_{\left(N_{x}, y-1\right) \rightarrow\left(N_{x}, y\right)}^{*}+\sigma_{\left(N_{x}-1, y\right) \rightarrow\left(N_{x}, y\right)}^{*}+\sigma_{\left(N_{x}, y+1\right) \rightarrow\left(N_{x}, y\right)}^{*}\right)$.

Note here that contrary to the classical way of proceeding for SIP measurements, the electrical potential is imposed at the boundaries, not the electrical current. Eqs (4), (6a), (6b), (8a) and (8c), together with eqs (5), (8b) and (8d), form a linear system of equations with $N_{x} \cdot N_{y}$ unknowns $V_{x, y}^{*}$. For simplification, we divide all the $V_{x, y}^{*}$ in the equations by $V_{0} e^{i \omega t}$, defining the real numbers $f_{x, y}$ and $\phi_{x, y}$ by:

$\frac{V_{x, y}^{*}}{V_{0} e^{i \omega t}}=f_{x, y}+i \theta_{x, y}$.

Similarly, we introduce the real numbers $g_{p \rightarrow q}$ and $\gamma_{p \rightarrow q}$ as

$\sigma_{p \rightarrow q}^{*}=g_{p \rightarrow q}+i \gamma_{p \rightarrow q}$.

Doing so, the system can take the form:

$\left[\begin{array}{cc}\mathbf{G} & -\boldsymbol{\Gamma} \\ \boldsymbol{\Gamma} & \mathbf{G}\end{array}\right]\left[\begin{array}{l}\mathbf{f} \\ \boldsymbol{\theta}\end{array}\right]=\left[\begin{array}{l}\mathbf{z} \\ \zeta\end{array}\right]$,

where matrices $\mathbf{G}$ and $\boldsymbol{\Gamma}$ and vectors $\mathbf{z}$ and $\zeta$ are real.

System (11) is solved using the QR decomposition (qr function in Matlab) to obtain $\mathbf{f}$ and $\boldsymbol{\theta}$. Then, knowing $V_{x, y}^{*} / V_{0} e^{i \omega t}$ at each nodes (from eq. 9), it is possible to compute the complex electrical current in each tube using eq. (3).

\subsection{Amplitude and phase spectra of the network}

At the macroscopic scale, the total current $I^{*}$ through the network is related to the applied macroscopic potential gradient $\Delta V^{*}$ by Ohm's law $I^{*}=-\sigma_{\text {bulk }}{ }^{*} \Delta V^{*}$, where $\sigma_{\text {bulk }}{ }^{*}$ is the bulk conductivity of the network. As a result, the conductivity amplitude $\alpha$ for a given 
frequency pulsation $\omega$ (i.e. the wavelength of the imposed sinusoidal electrical potential at the boundaries) is given by:

$\left\{\begin{array}{l}\alpha_{\text {in }}(\omega)=\left|\frac{I_{\text {in }}{ }^{*}}{\Delta V^{*}}\right|=\left|\frac{J_{\text {in }}^{\text {total }}}{V_{0} e^{i \omega t}}\right|=\left|\frac{1}{V_{0} e^{i \omega t}} \sum_{x=1}^{N_{x}} J_{(x, 1) \rightarrow(x, 2)}\right| \\ \alpha_{\text {out }}(\omega)=\left|\frac{I_{\text {out }}}{\Delta V^{*}}\right|=\left|\frac{J_{\text {ott }}^{\text {tot }}}{V_{0} e^{i \omega t}}\right|=\left|\frac{1}{V_{0} e^{i \omega t}} \sum_{x=1}^{N_{x}} J_{\left(x, N_{y}-1\right) \rightarrow\left(x, N_{y}\right)}\right|,\end{array}\right.$

and the phase shift $\varphi$ by:

$$
\left\{\begin{aligned}
\varphi_{\text {in }}(\omega) & =\arg \left(-\frac{I_{\text {in }}{ }^{*}}{\Delta V^{*}}\right)=\arg \left(V_{0} e^{i \omega t}\right)-\arg \left(J_{\text {in }}^{\text {total }}\right) \\
& =-\arg \left(\sum_{x=1}^{N_{x}} J_{(x, 1) \rightarrow(x, 2)}\right) \\
\varphi_{\text {out }}(\omega) & =\arg \left(-\frac{I_{\text {out }}{ }^{*}}{\Delta V^{*}}\right)=\arg \left(V_{0} e^{i \omega t}\right)-\arg \left(J_{\text {out }}^{\text {total }}\right) \\
& =-\arg \left(\sum_{x=1}^{N_{x}} J_{\left(x, N_{y}-1\right) \rightarrow\left(x, N_{y}\right)}\right)
\end{aligned}\right.
$$

As the total complex current is conservative in the low-frequency limit of the Maxwell equations, in which we work, we have $\alpha_{\text {in }}=\alpha_{\text {out }}$ and $\varphi_{\text {in }}=\varphi_{\text {out }}$. In practice, the computations produce values having a relative difference lower than $1 \times 10^{-10}$ per cent.

\subsection{Local model for the complex resistivity}

For the complex resistivity of each tube linking node $p$ to node $q$, denoted $\rho_{p \rightarrow q}^{*}$, we arbitrarily used a 'local' Pelton resistivity model (Pelton et al. 1978; see also Tarasov \& Titov 2013):

$\rho_{p \rightarrow q}^{*}=\rho_{0, l}\left(1-m_{l}\left(1-\frac{1}{1+\left(i \omega \tau_{p \rightarrow q}\right)^{c_{l}}}\right)\right)=\frac{1}{\sigma_{p \rightarrow q}^{*}}$.

Here $\rho_{0, l}$ corresponds to the DC resistivity (in Ohm·m), $m_{l}$ is the chargeability (in $\mathrm{V} \cdot \mathrm{V}^{-1}$ - dimensionless) equal to $\left(\rho_{0, l}-\rho_{\infty, l}\right) / \rho_{0, l}$, where $\rho_{\infty, l}$ is the limit of $\rho_{p \rightarrow q}^{*}$ for infinite frequency, $\tau_{p \rightarrow q}$ is the time constant (in s) and $c$ the so-called Cole-Cole exponent (dimensionless) that is comprised between 0 and 1 . The Pelton model, a derivation from the Cole-Cole model, was originally designed to explain macroscopic IP spectra. However, it has been demonstrated to be a good model to represent the polarization of single grains or pores (Leroy et al. 2008; Niu \& Revil 2016).

In eq. (14), $\tau_{p \rightarrow q}$ is linked to the radius of the tube $r_{p \rightarrow q}$ by:

$\tau_{p \rightarrow q}=\frac{r_{p \rightarrow q}^{2}}{2 D_{l}}$,

$D_{l}$ being the diffusion coefficient of the counterions in the Stern layer (Revil et al. 2012; Niu \& Revil 2016). Eq. (15) is valid for a sequence of at least two tubes in membrane polarization models (e.g. Titov et al. 2002; Bücker \& Hördt 2013a,b), and also in the realm of the dynamic Stern layer model (Niu \& Revil 2016). It is important to underline here that the values $\rho_{0, l}, m_{l}, c_{l}$ and $D_{l}$ are the same for all the tubes. We recognize that the fact that $\rho_{0, l}$ and $m_{l}$ are the same for all tubes is a strong hypothesis: in reality, pores can present variations of these parameters, depending for example on the content of coating clays. This approach was adopted to simplify the problem, in order to have only one varying parameter (i.e. the radius).

\subsection{Upscaling summary}

To summary, the direct upscaling procedure for a given frequency consists in:

(1) Attributing a complex resistivity to each tube of the network.
(2) Obtaining the electrical potential at each node by solving the conservation laws.

(3) Computing the resulting total electrical current inflowing or outflowing from the network.

(4) Deducing the amplitude and phase value from this current.

\section{APPLICATION TO SYNTHETIC PORE RADIUS DISTRIBUTIONS}

\subsection{Principle}

For a given tube radius distribution, a random network is generated. To have a sufficiently representative medium (i.e. larger than the elementary representative volume, see end of Section 3.2), we use a $100 \times 100$ mesh (i.e. 19800 tubes). Then the macroscopic (i.e. the bulk) IP response of this network, in terms of the resistivity (or, equivalently, conductivity) amplitude and phase, is computed, for a given set of frequencies $f=\omega /(2 \pi)$. Note that IP measurements are typically performed in the frequency range from $1 \mathrm{mHz}$ to $20 \mathrm{kHz}$, but here we restrain the upper boundary to $1 \mathrm{kHz}$.

For all the tests reported in this section, we choose the 'local' values $\rho_{0, l}=100 \mathrm{Ohm} \cdot \mathrm{m}, m_{l}=0.1 \mathrm{~V} \cdot \mathrm{V}^{-1}, c_{l}=0.5$ and $\log 10\left(D_{l}\right)=-11$ in eqs (14) and (15), based on preliminary tests carried out on real samples (see Section 4). We choose the local value $c_{l}=0.5$ based on the recent works by Revil et al. (2014a) and Niu \& Revil (2016); this is representative of a diffusive process (elementary Warburg model, see Revil et al. 2014a). Note that in real rocks, $\rho_{0, l}$ and $m_{l}$ are expected to vary from pore to pore (e.g. depending on the content of coating clays; moreover, $\rho_{0, l}$ should be linked to the radius of the pore: the surface conductivity, and thus the total conductivity, increases with decreasing radius for instance). We choose them as constant here only to discuss the upscaling effect associated with the pore size distribution, therefore simplifying the physics of the problem.

Finally, we fit the macroscopic IP response of the network also by Pelton resistivity model, that is, the bulk resistivity $\rho^{*}$ bulk writes:

$\rho_{\text {bulk }}^{*}=\rho_{0, m}\left(1-m_{m}\left(1-\frac{1}{1+\left(i \omega \tau_{m}\right)^{c_{m}}}\right)\right)=\frac{1}{\sigma_{\text {bulk }}^{*}}$,

since, as discussed in several papers (Revil et al. 2014a,b, 2015; Niu \& Revil 2016), clean sandstones as well as clay-coated sandstones would exhibit such a behaviour.

We search for the macroscopic parameters $\rho_{0, m}, m_{m}, c_{m}$ and $\log _{10}\left(\tau_{m}\right)$ producing a Pelton resistivity model that fits the best the computed macroscopic IP response-what we call 'macroscopic optimization'. We use simulated annealing (Aarts \& Van Laarhoven 1985) as optimization procedure. A description of the algorithm can be found in Maineult (2016) — but without the monotony test. The variation ranges are $\left[\begin{array}{ll}80 & 120\end{array}\right] \mathrm{Ohm} \cdot \mathrm{m}$ for $\rho_{0, m},\left[\begin{array}{ll}0 & 1\end{array}\right]$ for $m_{m}$ and $c_{m}$, and $\left[\begin{array}{ll}-3 & 3\end{array}\right]$ for $\log _{10}\left(\tau_{m}\right)$. This first optimization is then followed by a simplex optimization (Nelder \& Mead 1965) to refine the solution (function fminsearch in Matlab). Finally, we estimate the macroscopic parameter $D_{m}$ using

$D_{m}=\frac{r_{\text {peak }}^{2}}{2 \tau_{m}}$,

where $r_{\text {peak }}$ is the radius associated with the maximum of the radius distribution. 

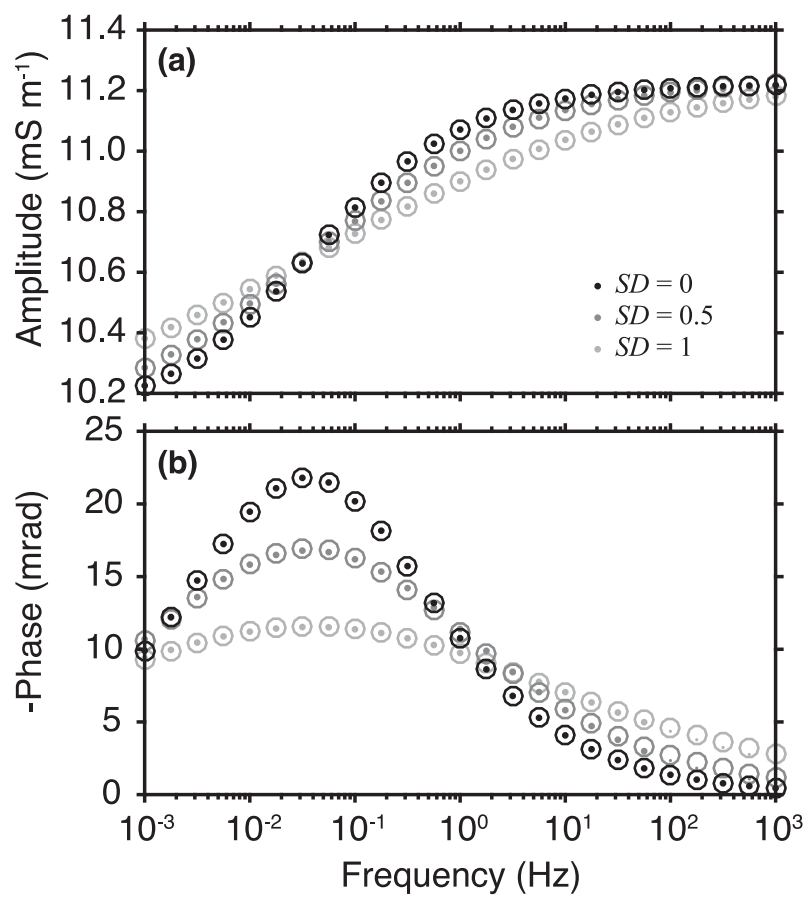

Figure 3. IP response of random uncorrelated media (mean radius $10 \mu \mathrm{m}$, standard deviation of $0,0.5$ and 1 , respectively), for the local parameters $\rho_{0, l}=100 \mathrm{Ohm} \cdot \mathrm{m}, m_{l}=0.1 \mathrm{~V} \cdot \mathrm{V}^{-1}, \log _{10}\left(D_{l}\right)=-11$ and $c_{l}=0.5$. The dots correspond to the computed response of the networks ('synthetic data'), the circles to the predictions resulting from the macroscopic optimization of the synthetic data. Note that the smaller dots correspond to synthetic data that were not taken into account for the optimization.

\subsection{Monomodal radius distribution, uncorrelated media}

We first study uncorrelated media (see Fig. 2b for an example). The radii $r$ are distributed in such a way that their decimal logarithms are normally distributed, that is

$P\left(\log _{10}(r) \leq X\right)=\frac{1}{2}+\frac{1}{2} \operatorname{erf}\left(\frac{X-\log _{10}\left(r_{\text {peak }}\right)}{\mathrm{SD} \sqrt{2}}\right)$,

where SD is the standard deviation; we set $r_{\text {peak }}$ equal to $10 \mu \mathrm{m}$ (see Fig. 2a for an example). In order to study the effect of the increasing heterogeneity of the radius distribution (i.e. its broadening), we start from the Dirac distribution (i.e. the standard deviation equal to 0 , corresponding to a homogeneous medium with all radii set to $10 \mu \mathrm{m})$, and then increase the standard deviation until 1 .

Three examples of computed spectra are shown in Fig. 3 (dots), for $\mathrm{SD}=0,0.5$ (corresponding to the network shown in Fig. 2) and 1 . The amplitudes increase from around 10.3 to $11.2 \mathrm{mS} \mathrm{m}^{-1}$ (Fig. 3a). The phase reaches a maximum value of about $22 \mathrm{mrad}$ at $31.6 \mathrm{mHz}$ for the Dirac distribution. Increasing standard deviation lowers the value of the phase peak and broadens the spectrum, but the peak frequency remains the same (Fig. 3b).

The predictions of the macroscopic optimization explain very well the computed IP responses (circles in Fig. 3), meaning that the macroscopic IP response of the networks obeys a Pelton resistivity model effectively (eq. 16). The estimated macroscopic parameters resulting from the macroscopic optimization are given in Table 1. Note that $\rho_{0, m}$ is equal to 99 , not $100 \mathrm{Ohm} \cdot \mathrm{m}$. This results from the size of the network: smaller networks produce values lower than 99, whereas larger ones produce values tending towards 100 (for instance, $10 \times 10$ networks give $90 \mathrm{Ohm} \cdot \mathrm{m}, 25 \times 2596$, and 50
Table 1. Macroscopic model parameters for the random uncorrelated media.

\begin{tabular}{lcccc}
\hline Standard deviation & $\rho_{0, m}$ & $m_{m}$ & $\log _{10}\left(D_{m}\right)$ & $c_{m}$ \\
\hline 0.00 & 99.0000 & 0.10000 & -11.0000 & 0.50000 \\
0.05 & 99.0034 & 0.10005 & -11.0006 & 0.49820 \\
0.10 & 99.0130 & 0.10019 & -11.0018 & 0.49291 \\
0.25 & 99.0641 & 0.10099 & -11.0008 & 0.46110 \\
0.50 & 99.2000 & 0.10323 & -11.0127 & 0.38427 \\
0.75 & 99.2723 & 0.10496 & -11.0228 & 0.31472 \\
1.00 & 99.3292 & 0.10618 & -11.0215 & 0.25974 \\
\hline
\end{tabular}

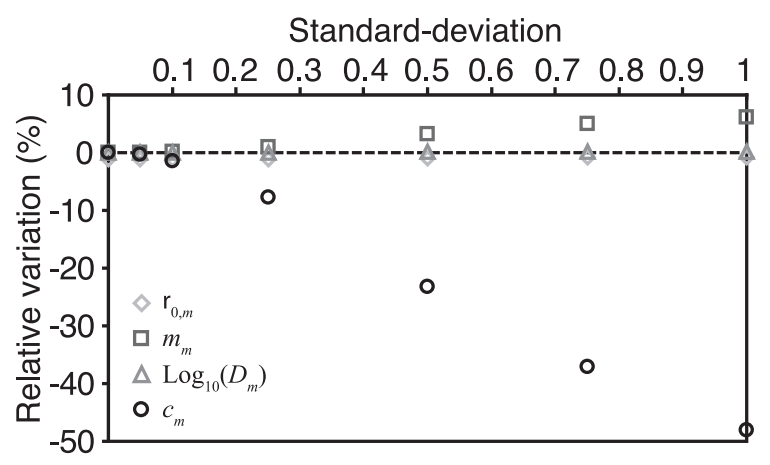

Figure 4. Relative differences between the macroscopic model parameters and the local model parameters (i.e. $\left(X_{m}-X_{l}\right) / X_{l}$, where $X$ is the considered parameter, $m$ standing for 'macroscopic' and $l$ for 'local') for random uncorrelated media, with respect to the standard deviation of the radius distribution. Local parameters are $\rho_{0, l}=100 \mathrm{Ohm} \cdot \mathrm{m}, m_{l}=0.1 \mathrm{~V} \cdot \mathrm{V}^{-1}$, $\log _{10}\left(D_{l}\right)=-11$ and $c_{l}=0.5$. Only the parameter $c_{m}$ varies significantly (up to -50 per cent).

$\times 50$ 98). However, networks larger than $100 \times 100$ nodes have huge computational time and can even cause memory overflow on a standard personal computer. When considering the increase of the standard deviation $\mathrm{SD}$, we observe that $\rho_{0, m}, m_{m}$, and $\log _{10}\left(D_{m}\right)$ are very stable, with low relative difference (Fig. 4 ), and are very close to $\rho_{0, l}, m_{l}$ and $\log _{10}\left(D_{l}\right)$. Interestingly, $c_{m}$ is very close to $c_{l}$ for very low standard deviations ( $\mathrm{SD}<0.1$ ), but diminishes significantly with increasing the network heterogeneity (up to -50 per cent of relative difference for $\mathrm{SD}=1$ ).

\subsection{Monomodal radius distribution, correlated media}

We then proceed similarly for correlated networks, which are more representative of real porous media. Indeed, in real media, the succession of pores is not random but obeys to some correlation length scales. We design our correlated network using an isotropic 2-D Von Karman correlation function defined by the standard deviation, $\sigma$, the Hurst exponent, $v$, whose value is comprised between 0 and 1 , and an isotropic characteristic length $L_{c}$. The Hurst exponent is directly related to the fractal dimension of the medium (e.g. Turcotte 1997). The 2-D power spectrum of the pore distribution may be usually normalized (Holliger \& Levander 1992) as:

$P\left(k_{x}, k_{y}\right)=\frac{\Gamma(v+1)}{\Gamma(v)} \frac{4 \pi \sigma^{2} L_{c}^{2}}{\left(1+k^{2}\right)^{v+1}}$,

with

$k^{2}=L_{c}^{2}\left(k_{x}^{2}+k_{y}^{2}\right)$. 
(a)

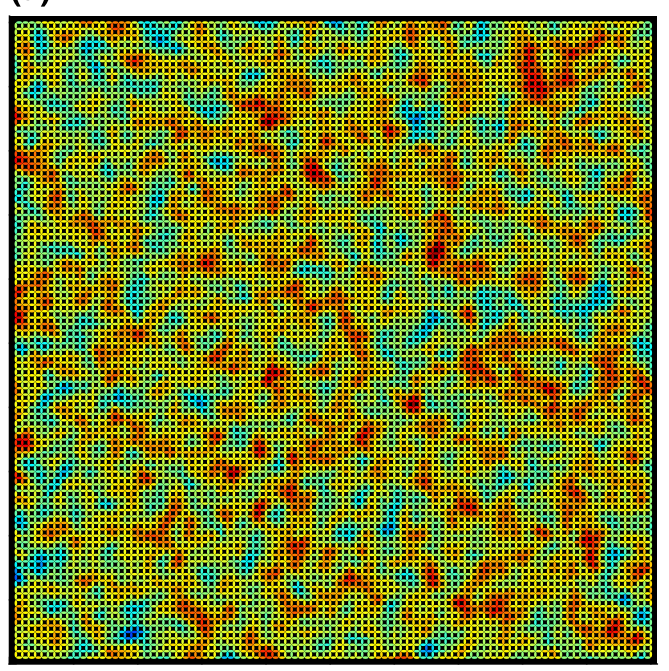

(b)

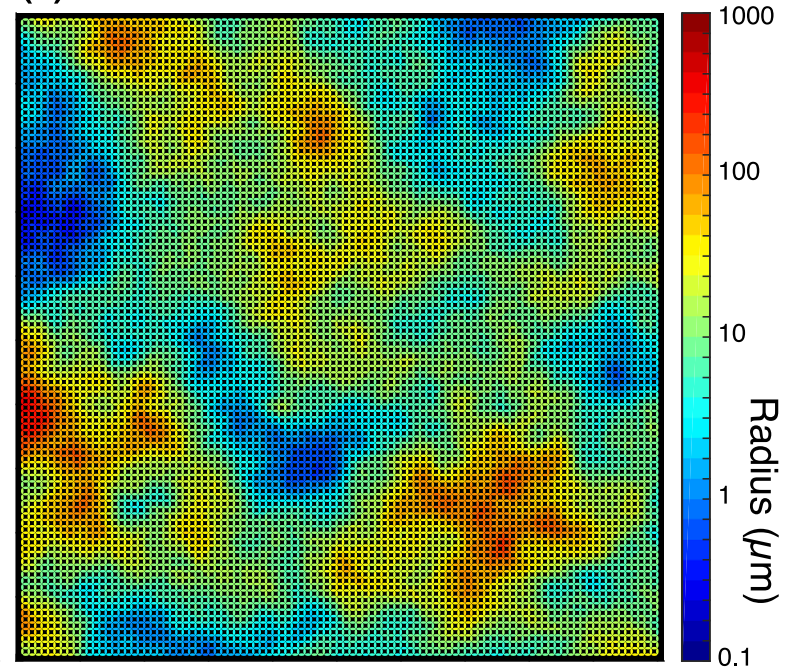

Figure 5. Example of random correlated media ( $100 \times 100$ nodes, 19800 tubes). The decimal logarithm is normally distributed, with a mean radius of $10 \mu \mathrm{m}$ and a standard deviation of 0.5 , a Hurst exponent of 0.9 and a characteristic scale $L_{c}$ of 1 (a) and 10 (b).

Table 2. Macroscopic model parameters for the random correlated media (Hurst exponent 0.9 and characteristic scale 1 ).

\begin{tabular}{lcccc}
\hline Standard deviation & $\rho_{0, m}$ & $m_{m}$ & $\log _{10}\left(D_{m}\right)$ & $c_{m}$ \\
\hline 0.0000 & 99.0000 & 0.10000 & -11.0000 & 0.50000 \\
0.0501 & 99.0034 & 0.10005 & -11.0005 & 0.49819 \\
0.1025 & 99.0139 & 0.10020 & -11.0016 & 0.49258 \\
0.2510 & 99.0600 & 0.10095 & -11.0085 & 0.46122 \\
0.4942 & 99.2025 & 0.10320 & -11.0283 & 0.38583 \\
0.7542 & 99.2746 & 0.10487 & -11.0325 & 0.31443 \\
1.0038 & 99.4226 & 0.10692 & -11.0785 & 0.26005 \\
\hline
\end{tabular}

Here, $k_{x}$ and $k_{y}$ are the wavenumber components and $\Gamma$ is the gamma function. A random mesh of 'virtual' radius values, with a size of $N_{x} \cdot N_{y}$, was then generated in the spectral domain and transformed in the spatial domain by a 2-D inverse Fourier transform of the square root of the power spectrum multiplied by a random phase between $-\pi$ and $\pi$ (Bohlen 2002). Finally, the value of the radius of the tube between node $p$ and $q$ is taken as the mean of the values of the 'virtual' radii at node $p$ and $q$, respectively. At the end, we check that the peak radius of the so-obtained tube radius distribution, as well as its standard deviation SD, are effectively equal to the desired peak radius value and standard deviation.

Two examples of correlated networks, with a radius distribution having a peak radius of $10 \mu \mathrm{m}$ and a standard deviation of 0.5 , are shown in Fig. 5. The Hurst exponent is equal to 0.9. The characteristic length size $L_{c}$ defines the size of the aggregates $\left(L_{c}=1\right.$ in Fig. 5a and 10 in Fig. 5b). The results of the macroscopic optimization (Tables 2 and 3 ) yield to the same conclusions as before, that is to say $\rho_{0, m}, m_{m}$ and $\log _{10}\left(D_{m}\right)$ do not vary significantly with increasing standard deviation. At the opposite, the values of $c_{m}$ decrease exactly as for the uncorrelated network cases (Fig. 6).

\subsection{Bimodal radius distribution, uncorrelated media}

Finally, we study uncorrelated media having a radius distribution with two peaks (similar, for instance, to rocks exhibiting a double porosity, such as some limestones, or sandstones with high clay
Table 3. Macroscopic model parameters for the random correlated media (Hurst exponent 0.9 and characteristic scale 10).

\begin{tabular}{lcccc}
\hline Standard deviation & $\rho_{0, m}$ & $m_{m}$ & $\log _{10}\left(D_{m}\right)$ & $c_{m}$ \\
\hline 0.0000 & 99.0000 & 0.10000 & -11.0000 & 0.50000 \\
0.0510 & 99.0032 & 0.10005 & -11.0001 & 0.49813 \\
0.1079 & 99.0151 & 0.10022 & -11.0012 & 0.49179 \\
0.2489 & 99.0604 & 0.10096 & -11.0074 & 0.46151 \\
0.4944 & 99.2981 & 0.10388 & -11.0679 & 0.38305 \\
0.8096 & 99.2408 & 0.10413 & -11.0497 & 0.30667 \\
1.0299 & 99.5149 & 0.10554 & -11.2612 & 0.26588 \\
\hline
\end{tabular}

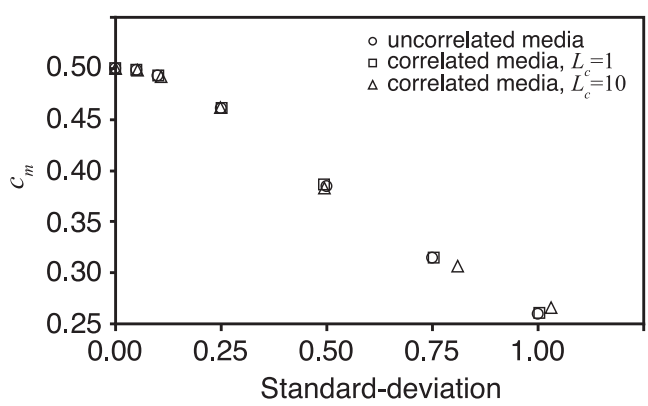

Figure 6. Evolution of the macroscopic model parameter $c_{m}$ with respect to the standard deviation, for the random uncorrelated and random correlated media. Local parameters are $\rho_{0, l}=100 \mathrm{Ohm} \cdot \mathrm{m}, m_{l}=0.1 \mathrm{~V} \cdot \mathrm{V}^{-1}$, $\log _{10}\left(D_{l}\right)=-11$ and $c_{l}=0.5$.

content). We use the following cumulative probability:

$$
\begin{aligned}
P\left(\log _{10}(r) \leq X\right)= & \frac{1}{2}+\frac{1}{4} \operatorname{erf}\left(\frac{X-\log _{10}\left(r_{\text {peak }, 1}\right)}{\mathrm{SD} \sqrt{2}}\right) \\
& +\frac{1}{4} \operatorname{erf}\left(\frac{X-\log _{10}\left(r_{\text {peak }, 2}\right)}{\mathrm{SD} \sqrt{2}}\right) .
\end{aligned}
$$

Note that we give the same weight to the two peaks in order to restrain the number of varying parameters.

Examples of studied distributions, for $r_{\text {peak, } 1}=10 \mu \mathrm{m}$ and $r_{\text {peak }, 2}=0.1 \mu \mathrm{m}$, are shown in Fig. 7 , and associated IP spectra in Fig. 8. For the double Dirac distribution $(\mathrm{SD}=0$, Fig. $7 \mathrm{a})$, we 


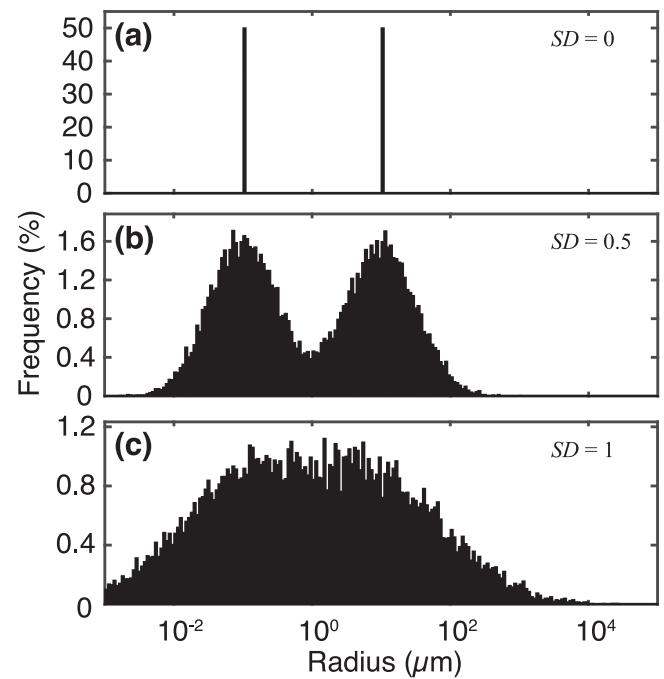

Figure 7. Radius distributions for the random uncorrelated media with two peaks (mean radii 0.1 and $10 \mu \mathrm{m}$ with weight $1 / 2$ each, standard deviation of $0,0.5$ and 1 , respectively). For the standard deviation 1 , the two peaks overlap, explaining the observed unique peak in the IP response (see Fig. 8).
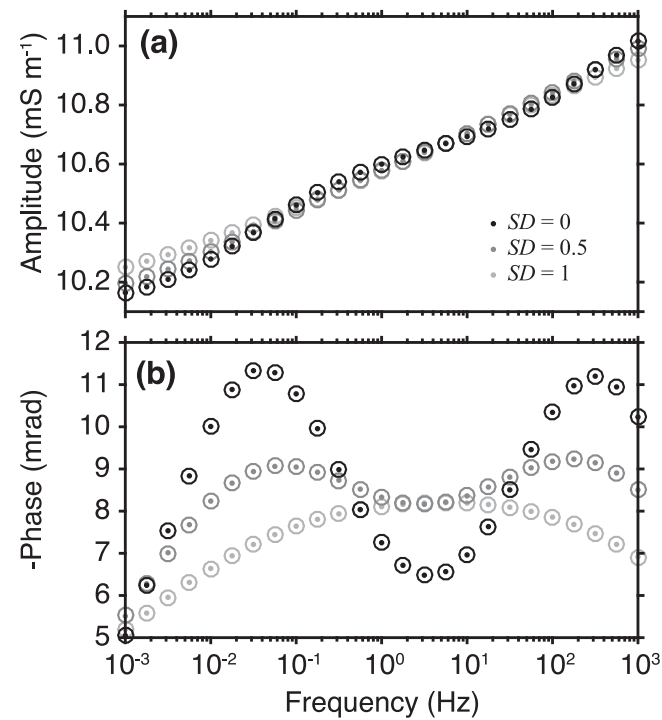

Figure 8. IP response of random uncorrelated media, with a two-peak radius distribution (mean radii 0.1 and $10 \mu \mathrm{m}$ with weight $1 / 2$ each, standard deviation of $0,0.5$ and 1 , respectively). Local parameters are $\rho_{0, l}=100 \mathrm{Ohm} \cdot \mathrm{m}$, $m_{l}=0.1 \mathrm{~V} \cdot \mathrm{V}^{-1}, \log _{10}\left(D_{l}\right)=-11$ and $c_{l}=0.5$. The dots correspond to the computed response of the networks ('synthetic data'), the circles to the predictions resulting from the macroscopic optimization of the synthetic data. The associated radius distributions are shown in Fig. 7.

can observe two inflexion points in the amplitude (black dots in Fig. 8a), and two peaks in the phase spectra (black dots in Fig. 8b). As evidenced by the response of the distribution with $\mathrm{SD}=0.5$ (Fig. 7a), the increase in heterogeneity results in less marked inflexion points (dark grey dots in Fig. 8a) and in a decrease of the amplitude of the two-phase peaks (dark grey dots in Fig. 8b). When the two peaks of the radius distribution overlap (for instance for $\mathrm{SD}=1$, Fig. $7 \mathrm{c}$ ), the phase spectrum presents only one peak, but significantly broadened (light grey dots in Fig. 8b).
To find the macroscopic parameters of the networks, we fitted the macroscopic response with a dual Pelton resistivity model (e.g. Kemna et al. 2000):

$$
\begin{aligned}
\rho_{\text {bulk }}{ }^{*}= & \frac{1}{\sigma_{\text {bulk }}{ }^{*}}=\frac{\rho_{0, m, 1}}{2}\left(1-m_{m, 1}\left(1-\frac{1}{1+\left(i \omega \tau_{m, 1}\right)^{c_{m, 1}}}\right)\right) \\
& +\frac{\rho_{0, m, 2}}{2}\left(1-m_{m, 2}\left(1-\frac{1}{1+\left(i \omega \tau_{m, 2}\right)^{c_{m, 2}}}\right)\right),
\end{aligned}
$$

where subscript 1 (respectively 2) corresponds to parameters associated with $r_{\text {peak }, 1}$ (respectively $r_{\text {peak }, 2}$ ). The optimization procedure is the same as before. The predictions inferred from the estimated macroscopic parameters (circles in Fig. 8) fit very well the computed IP responses, meaning that the assumption of the dual Pelton resistivity model (eq. 22) was correct. The estimated macroscopic parameters (Table 4) have the same behaviour as observed for onepeak distributions, that is, only $c_{m, 1}$ and $c_{m, 2}$ varies significantly, decreasing with the increasing standard deviation of the tube radius distribution, as soon as SD is larger than 0.1 .

\section{APPLICATION TO REAL SAMPLES}

\subsection{Estimation of the parameters}

To study synthetic radius distributions (Section 3 ), we impose the local parameters of the Pelton complex resistivity $\left(\rho_{0, l}, m_{l}, c_{l}\right.$ and $D_{l}$ ), then compute the macroscopic response of the network, and finally fit this macroscopic response with a Pelton resistivity model to obtain the macroscopic parameters of the network $\left(\rho_{0, m}, m_{m}, c_{m}\right.$ and $D_{m}$ ). The procedure is slightly different for real samples, as the local parameters are here unknown.

To estimate the local parameters, we first construct a network $(25 \times 25$ nodes and 1200 tubes $)$ whose radius distribution obeys the measured pore access radius distribution inferred from mercury porosimetry (an example of such a distribution is shown in Fig. 9). Using the simulated annealing, we explore the parameter space to find $\rho_{0, l}, m_{l}, c_{l}$ and $D_{l}$ to be used in eqs (14) and (15) in order to obtain a macroscopic IP response of the network as close as possible to the measured IP response. Since a single network realization, especially on such small networks, have no statistical significance, we then explore again the parameter space for 100 network realizations with the simplex method, using as starting point the four local parameters resulting from the simulated annealing optimization on the initial network. An example of results from such a procedure is shown in Fig. 10: the crosses represent the measured data and the black lines represent the computed responses of the 100 network realizations. Each realization explains rather well the observations. For the estimated values of the local parameters $\rho_{0, l}, m_{l}, c_{l}$ and $D_{l}$, we use their mean values over the 100 realizations. Histograms of these local parameter values (Fig. 11) show that they are not very dispersed, and rather well determined. Finally, to obtain the macroscopic parameters $\rho_{0, m}, m_{m}, c_{m}$ and $D_{m}$, we fit the measured IP response with a Pelton resistivity model as before (Fig. 12, see Section 3.1).

\subsection{Results}

We use the data for the samples studied by Revil et al. (2014a), that is, six siliciclastic (sandstones and mudstones) samples from the Great Divide Basin (Wyoming, USA), denoted S436, S439, S490, S493, S498 and S499. The petrophysical properties of these materials are extensively described in Revil et al. (2014a) and will 
Table 4. Macroscopic model parameters for the random uncorrelated media with two peaks in the radius distribution. Subscript 1 corresponds to the $10 \mu \mathrm{m}$ peak, and subscript 2 to the $0.1 \mu \mathrm{m}$ peak.

\begin{tabular}{lcrcccccc}
\hline Standard deviation & $\rho_{0, m, 1}$ & \multicolumn{1}{c}{$\rho_{0, m, 2}$} & $m_{m, 1}$ & $m_{m, 2}$ & $\log _{10}\left(D_{m, 1}\right)$ & $\log _{10}\left(D_{m, 2}\right)$ & $c_{m, 1}$ & $c_{m, 2}$ \\
\hline 0.00 & 99.6590 & 98.3413 & 0.10282 & 0.09715 & -10.9773 & -10.9769 & 0.49989 & 0.49991 \\
0.05 & 98.3659 & 99.6393 & 0.10268 & 0.09741 & -10.9770 & -10.9802 & 0.49788 & 0.49811 \\
0.10 & 99.1972 & 98.8224 & 0.10142 & 0.09879 & -10.9801 & -10.9797 & 0.49194 & 0.49291 \\
0.25 & 97.8918 & 100.2166 & 0.10646 & 0.09522 & -10.9735 & -10.9748 & 0.45626 & 0.45793 \\
0.50 & 99.5091 & 98.8213 & 0.10284 & 0.10485 & -11.0293 & -11.0115 & 0.37106 & 0.35970 \\
0.75 & 99.6946 & 98.7478 & 0.09298 & 0.11975 & -11.1166 & -11.1103 & 0.30604 & 0.27599 \\
\hline
\end{tabular}

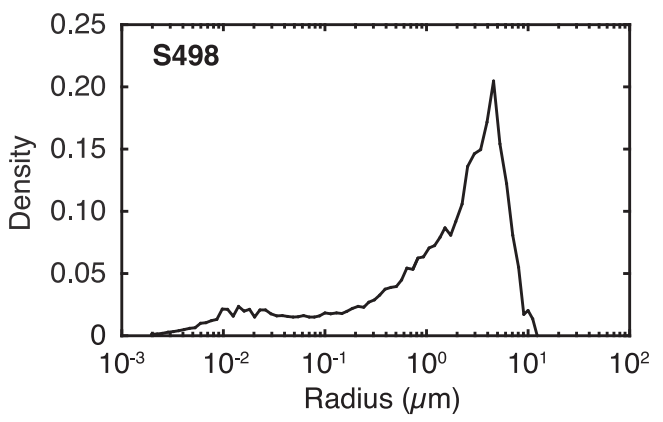

Figure 9. Pore radius distribution for core sample S498, inferred from mercury porosimetry measurements.

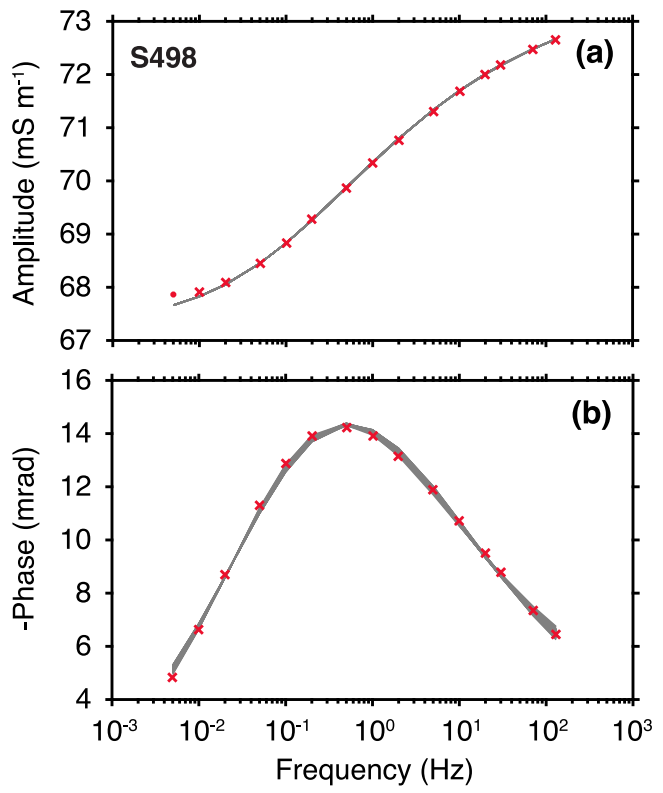

Figure 10. Measured IP response of core sample S498 (red crosses and dots), and the predictions resulting from the local optimization of these data (grey lines), carried out on 100 uncorrelated networks $(25 \times 25$ nodes, 1200 tubes). Note that the data figured by dots were not taken into account for inversion.

not be repeated here. Their pore size distributions are characterized from mercury porosimetry.

The local and macroscopic parameters are compared in Table 5 and in Fig. 13. It can be seen that $\rho_{0, l}$ and $\rho_{0, m}$ are very close (Fig. 13a), and the same for $m_{l}$ and $m_{m}$ (Fig. 13b). This behaviour is in agreement with what we observe on synthetic distributions. The relation between $\log _{10}\left(D_{l}\right)$ and $\log _{10}\left(D_{m}\right)$ is more dispersed (Fig. 13c), and does not follow the 1:1 trend. However, the absolute relative variations (computed as $2|(a-b) /(a+b)|)$ do not exceed 10 per cent (mean 5 per cent). Finally, $c_{m}$ is always smaller than $c_{l}$, as for the synthetic distributions, with absolute relative variations up to 48 per cent (mean 28 per cent, Fig. 13d).

\section{DISCUSSION}

The very simple methodology of random network produces coherent results, as evidenced by the response of the simple Dirac distribution corresponding to a homogeneous medium (Fig. 3). Under the assumption that the resistivity $\rho_{0, l}$, the chargeability $m_{l}$, the Cole-Cole exponent $c_{l}$ and the diffusion coefficient $D_{l}$ are the same for all the tubes (i.e. the only varying parameter is the tube radius), the macroscopic parameters (i.e. $\rho_{0, m}, m_{m}, c_{m}$ and $D_{m}$ ) are the same as the local ones (i.e. $\rho_{0, l}, m_{l}, c_{l}$ and $D_{l}$ ), as expected, meaning that the upscaling procedure is coherent and successful. Therefore, it appears that the random network methodology can be used as an efficient tool adequate for studying the upscaling of the IP problem, from the pore scale to the sample scale.

We observe, still under the assumption stated above, that using a Pelton resistivity model for the complex resistivity of the pores generates a Pelton resistivity model at the macroscopic scale, as shown in Fig. 3 (or a dual Pelton resistivity model in the case of bimodal radius distribution, Fig. 8). The macroscopic resistivity $\rho_{0, m}$, chargeability $m_{m}$ and diffusion coefficient $D_{m}$ are very close to the local resistivity $\rho_{0, l}$, chargeability $m_{l}$ and diffusion coefficient $D_{l}$, respectively (note here again that the local resistivity is not those of the fluid in the tube, but also includes the effects of the surrounding mineral). On the contrary, the macroscopic Cole-Cole exponent $c_{m}$ decreases with increasing heterogeneity, with relative difference $\left(c_{m}-c_{l}\right) / c_{l}$ up to -48 per cent for the standard deviation value equal to 1 when $c_{l}$ is equal to 0.5 (Fig. 4). We also computed the evolution of $c_{m}$ in function of the standard deviation SD for two supplemental cases: $c_{l}=0.25$ and $c_{l}=0.75$ (Fig. 14a). In the first case, the decrease is less marked, with maximal relative difference up to -25 percent, whereas in the second case the decrease is more important, with maximal relative difference up to -62 per cent (Fig. 14b). It means that the upscaling properties of the macroscopic Cole-Cole exponent depend also on the absolute value of the local Cole-Cole exponent, and not only on the heterogeneity of the porous medium.

This observation of a lower macroscopic value of the Cole-Cole exponent, made on synthetic pore radius distribution, is also valid for the few experimental measurements reported here (Fig. 13). From the upscaling point of view, and again under the assumptions we make, it could imply that the resistivity and chargeability values determined from measurements performed at the sample scale are representative of the values that they have at the pore scale. Note that the heterogeneity generates a strong variation in radius, and thus in the time constant $\tau$ (eq. 15). As the $\tau$ distribution is directly linked to the Cole-Cole exponent $c$ (Cole \& Cole 1941), this explains why $c$ varies so much with increasing heterogeneity. On the contrary, the local resistivity and chargeability were constant over the whole tube 

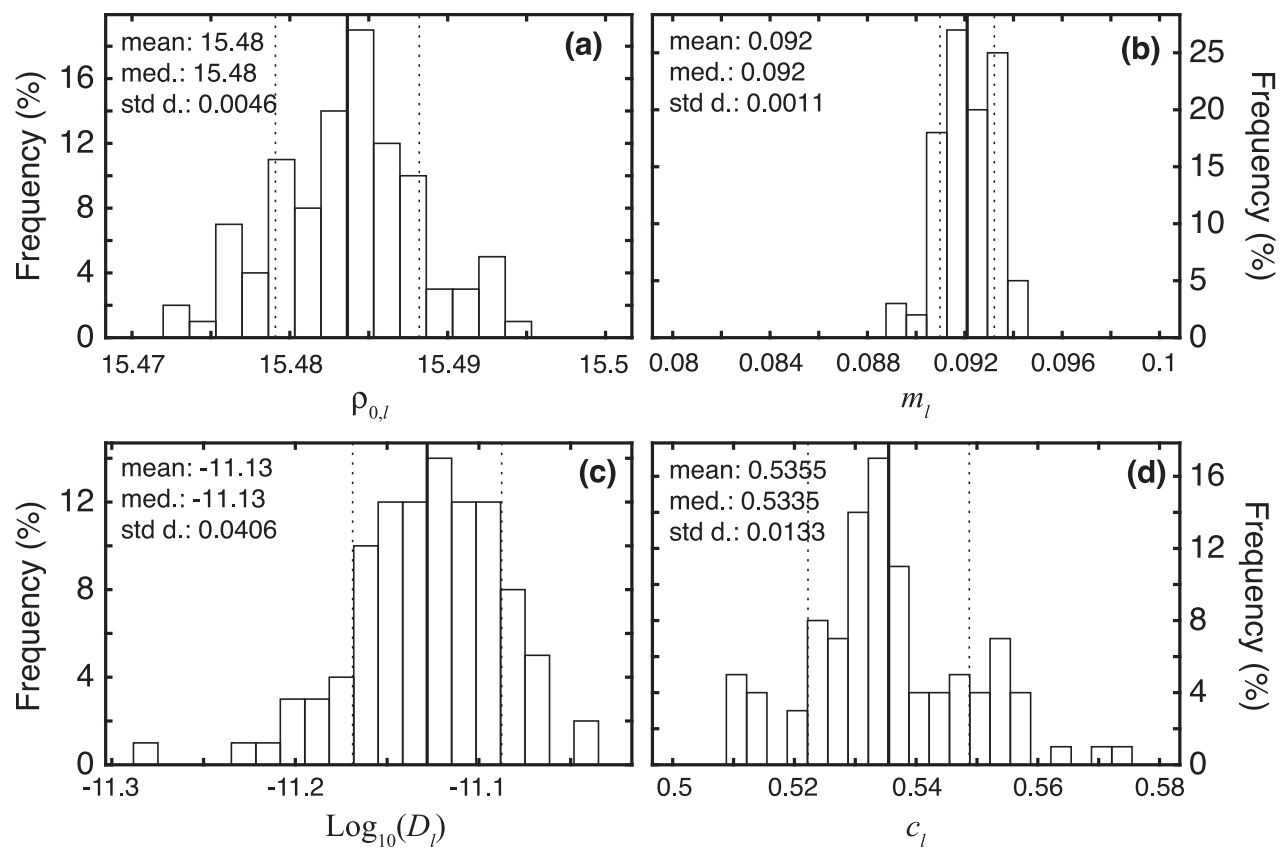

Figure 11. Distributions of the values of the local model parameters, resulting from the 100 local optimizations of the data for core sample S498.

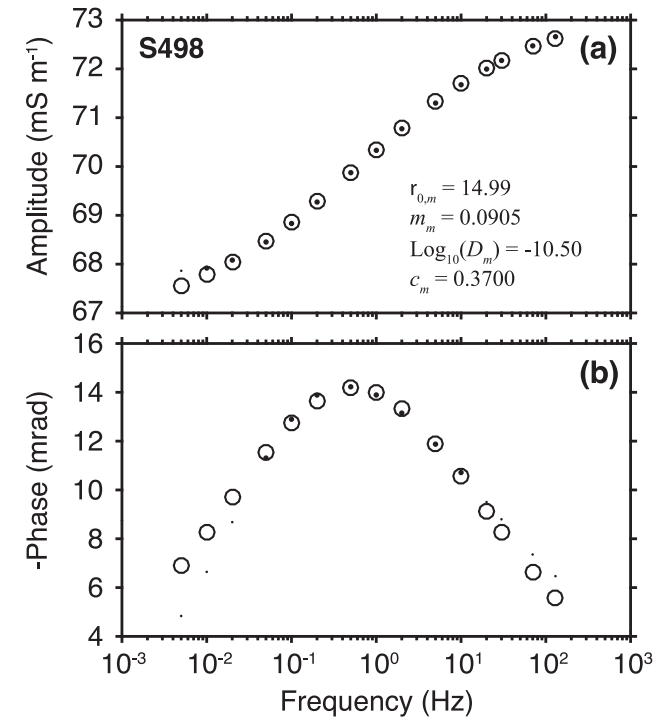

Figure 12. Measured IP response of core sample S498 (dots), and the predictions resulting from the macroscopic optimization of these data (circles). Note that the data figured by the smaller dots were not taken into account for the optimization.

network, resulting in very small variations at the macroscopic scale. Finally, we also observe that the macroscopic diffusion coefficient $D_{m}$ is systematically higher than the local coefficient $D_{l}$ (Fig. 13c), but the absolute relative variation as well as the number of samples are too small to conclude on the generalization of this observation. It may suggest that the macroscopic value of the time constant $\tau_{m}$ is slightly decreased compared to the average local value $\tau_{l}$ during the upscaling process.

Note also that the macroscopic parameters exhibit exactly the same behaviour for $25 \times 25$ and $50 \times 50$ networks (except the absolute value of the macroscopic resistivity, see Section 3.2). In particular, $c_{m}$ presents the same characteristics, that is, an identical decreasing rate with increasing standard deviation; but the results are much more erratic for $10 \times 10$ networks. It means that the elementary representative volume is larger than (or equal to) $25 \times 25$ and that the way $c_{m}$ is decreasing does not change with the increasing size of the network. In other words, the upscaling properties of the Cole-Cole exponent seem not to depend on the considered scale, provided that the considered volume is large enough.

Concerning the influence of the correlation distance of the radius distribution, it seems that the spatial repartition of the tube radii does not play a significant role on the evolution of the macroscopic parameters (still under the assumption stated above). The evolution of the Cole-Cole exponent $c_{l}$ is indeed the same in correlated and uncorrelated media (Fig. 4). The heterogeneity of the radius distribution is the most important parameter influencing the macroscopic IP response.

Introducing local distributions for the resistivity, chargeability and Cole-Cole exponent based on realistic scenarii constitutes the basis for future works. We preliminarily tested three simple cases. In the first one, the local resistivity $\rho_{0, \text { loc }}$ varies linearly with the varying radius, following $\rho_{0, \text { loc }}=100+50\left(\log _{10}(r)-\left\langle\log _{10}(r)\right\rangle\right)$ with $\rho_{0, \mathrm{loc}}$ in Ohm.m and $r$ in $\mu \mathrm{m}$ (Fig. 15a); as the radius decreases, the local resistivity diminishes since the stronger effect of the surface conductivity increases the pore conductivity. In the second one, the local chargeability $m_{\text {loc }}$ increases linearly with the decreasing radius, following $m_{\mathrm{loc}}=0.1+0.05\left(\left\langle\log _{10}(r)\right\rangle-\log _{10}(r)\right)$ with $m_{\text {loc }}$ in $\mathrm{V} \cdot \mathrm{V}^{-1}$ and $r$ in $\mu \mathrm{m}$ (Fig. 15b). Finally, the third case is the combination of the first and the second case. We observe that when the local resistivity varies, the conductivity amplitude spectrum (Fig. 16a) is shifted towards the higher conductivities, whereas the phase spectrum (Fig. 16b) does not change. When the local chargeability varies, the amplitude spectrum is slightly shifted towards lower conductivities at lower frequencies; the phase spectra is shifted toward the higher frequencies, meaning that the macroscopic time relaxation constant has decreased (Table 6). When both vary, we observe a combination of the two previous effects. Again, 
Table 5. Local (subscript « $1 »)$ and macroscopic (subscript « $\mathrm{m} »)$ model parameters for the samples.

\begin{tabular}{lrrllllll}
\hline Sample & \multicolumn{1}{c}{$\rho_{0, l}$} & \multicolumn{1}{c}{$\rho_{0, m}$} & \multicolumn{1}{c}{$m_{l}$} & \multicolumn{1}{c}{$m_{m}$} & $\log _{10}\left(D_{l}\right)$ & $\log _{10}\left(D_{m}\right)$ & $c_{l}$ & $c_{m}$ \\
\hline S436 & 51.0687 & 50.1435 & 0.23084 & 0.24139 & -11.8476 & -10.7534 & 0.35182 & 0.25442 \\
S439 & 9.6419 & 9.2862 & 0.034705 & 0.031959 & -10.9826 & -10.6858 & 0.765 & 0.48392 \\
S490 & 35.6619 & 34.5903 & 0.17737 & 0.16454 & -10.8596 & -10.3923 & 0.48814 & 0.37589 \\
S493 & 9.8835 & 9.5018 & 0.068381 & 0.059576 & -10.8382 & -10.4972 & 0.42828 & 0.37044 \\
S498 & 15.4836 & 14.9913 & 0.092097 & 0.090523 & -11.1282 & -10.4985 & 0.53548 & 0.37002 \\
S499 & 45.4526 & 43.9791 & 0.21947 & 0.19424 & -10.648 & -10.2643 & 0.49969 & 0.43049 \\
\hline
\end{tabular}
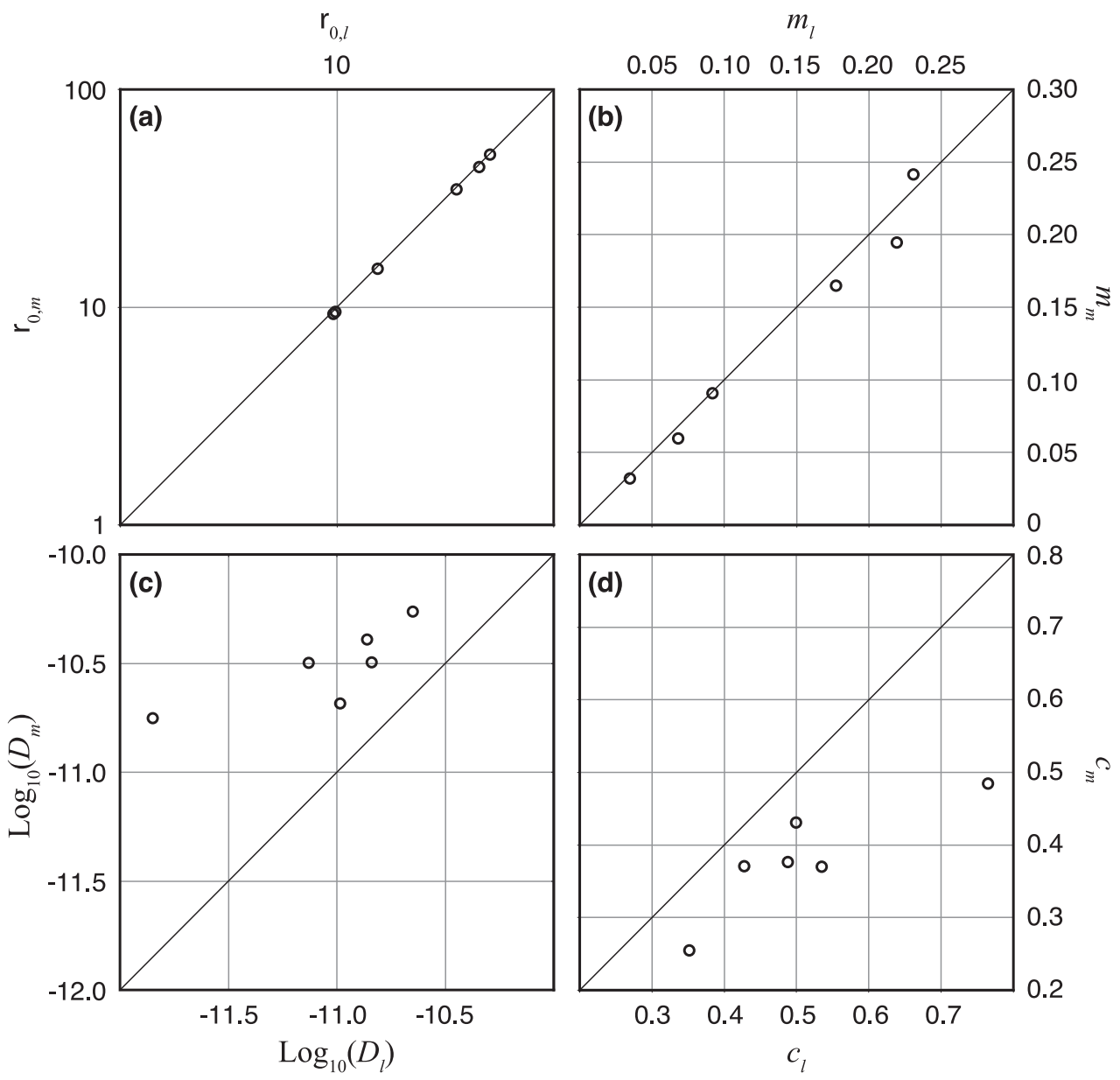

Figure 13. Comparison of the macroscopic and local model parameters: (a) resistivity $\rho_{0}$, (b) chargeability $m$, (c) diffusion coefficient $D$ and (d) Cole-Cole exponent $c$.

these simple examples evidenced the interest of the tube network for the study of the IP upscaling.

We can note here than the macroscopic spectra can also be inverted in terms of complex conductivity model, using:

$\sigma_{\text {bulk }}^{*}=\sigma_{0, m}\left(1+\frac{m_{m}^{\prime}}{1-m_{m}^{\prime}}\left(1-\frac{1}{1+\left(i \omega \tau_{m}^{\prime}\right)^{c^{\prime} m}}\right)\right)$

(see for instance Tarasov \& Titov 2013) instead of eq. (16). We should have $\sigma_{0, m}=\rho_{0, m}{ }^{-1}, m_{m}{ }^{\prime}=m_{m}, c_{m}{ }^{\prime}=c_{m}$ and:

$\tau_{m}^{\prime}=\left(1-m_{m}\right)^{1 / c} \tau_{m}$

(Florsch et al. 2010; Tarasov \& Titov 2013). For instance, when inverting the third case using the Pelton resistivity model, we obtained $\rho_{0, m}=95.65 \mathrm{Ohm} \cdot \mathrm{m}, m_{m}=0.104 \mathrm{~V} \cdot \mathrm{V}^{-1}, c_{m}=0.389$ and $\tau_{m}=2.9231$ (Table 6). Using the complex conductivity model, we get $1 / \sigma_{0, m}=95.65 \mathrm{Ohm} \cdot \mathrm{m}, m_{m}{ }^{\prime}=0.104 \mathrm{~V} \cdot \mathrm{V}^{-1}, c_{m}{ }^{\prime}=0.389$ and $\tau_{m}{ }^{\prime}=2.2054$. Eq. (24) is therefore verified, proving again the coherence of our approach.

Using the Pelton resistivity model for the complex resistivity at the pore scale may appear restrictive, since it somehow removes the question of the physical phenomena occurring at the pore scale. We would answer that we used the Pelton resistivity model exactly for this reason: we wanted to simplify the pore scale model and focus on the upscaling issue only. Further works obviously require the implementation of more physical responses at the pore scale (such as models by Titov et al. 2002; Revil \& Florsch 2010; Bücker \& Hördt 2013a,b), as well as the study of the influence of the pore geometry - that is, is this upscaling property still valid if pores are more complicated than simple tubes? An extension to 3-D networks is also possible to produce networks more representative of real core samples. Finally, the methodology we use opens other research directions, such as the study of the effect of anisotropy, which can be easily implemented in correlated networks, the extension to grain 

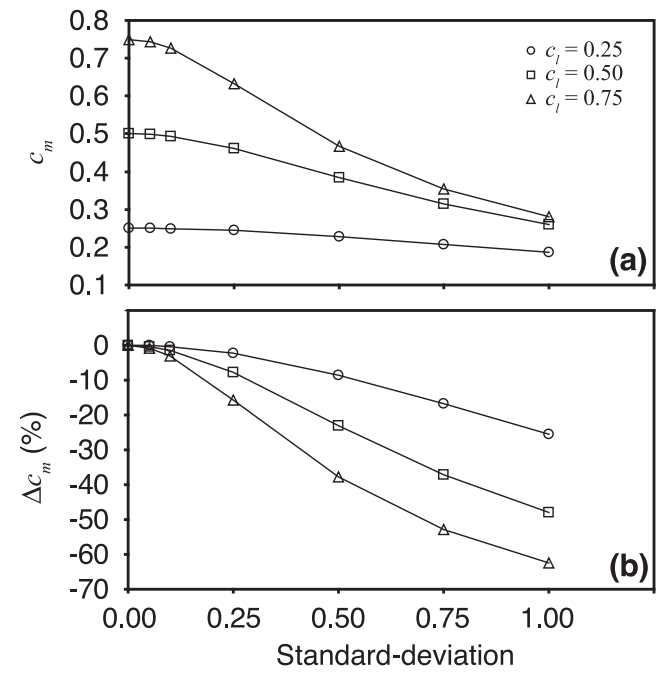

Figure 14. Evolution of the macroscopic model parameter $c_{m}$ with respect to the standard deviation for the (a) random uncorrelated media, and (b) associated relative difference $\left(c_{m}-c_{l}\right) / c_{l}$, for $c_{l}=0.25,0.5$ and 0.75 , respectively. Other local parameters are $\rho_{0, l}=100 \mathrm{Ohm} \cdot \mathrm{m}, m_{l}=0.1 \mathrm{~V} \cdot \mathrm{V}^{-1}$ and $\log _{10}\left(D_{l}\right)=-11$.

assemblages instead of pore assemblages, and even the study of drainage and imbibition with local models taking into account the effect of the saturation.

\section{CONCLUSIONS}

We implement the tube network methodology to study the upscaling of IP response from the pore scale (the local scale) to the sample scale (the macroscopic scale) on a very simplified system. Using a Pelton resistivity model for the complex resistivity of the tubes, with identical resistivity, chargeability, Cole-Cole exponent and diffusion coefficient values for all the tubes but a different time constant that depends on the radius, we show that the macroscopic response also obeys a Pelton resistivity model, with the same resistivity, chargeability and diffusion coefficient values, but with a different Cole-Cole exponent. The macroscopic value of this last parameter decreases strongly with the increasing heterogeneity of the pore radius distribution, for correlated and uncorrelated media. The same behaviour was observed on six sandstone samples.

We demonstrate the usefulness of the method for the study of the upscaling of IP response in the simplified case of a local Pelton resistivity model. Further works will require the implementation of more physical models for the IP properties at the pore scale, in order

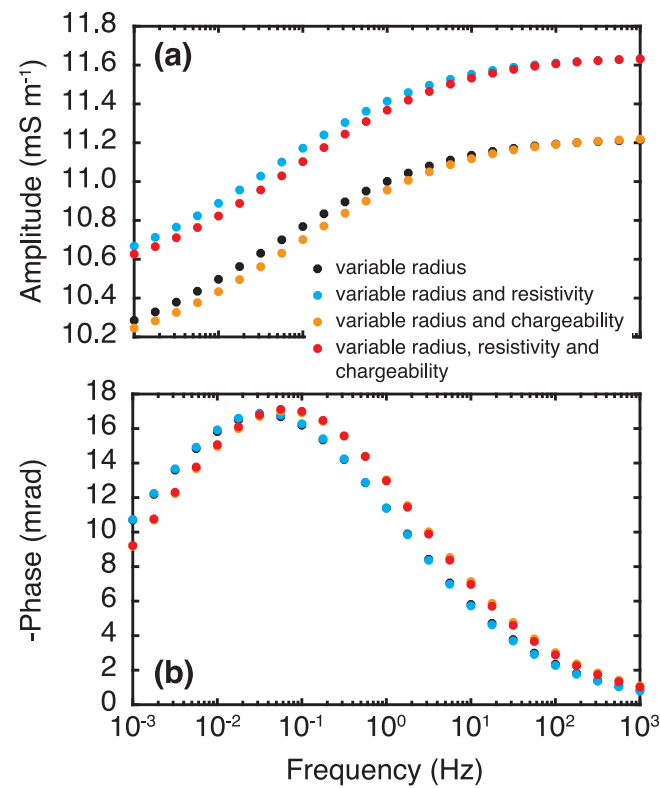

Figure 16. Macroscopic IP spectra obtained in the case of variable radius (black dots), variable radius and local resistivity (blue dots), variable radius and local chargeability (orange dots), and variable radius, local resistivity and local chargeability (red dots), using the relationships described in Fig. 15 for the case of the $100 \times 100$ random uncorrelated network with the decimal logarithm of the pore tube radius distribution normally distributed, with a mean radius of $10 \mu \mathrm{m}$ and a standard deviation of 0.5 (shown in Fig. 2b).

Table 6. Macroscopic model parameters for the spectra shown in Fig. 16, deduced by inversion.

\begin{tabular}{lcccc}
\hline Case & $\rho_{0, m}$ & $m_{m}$ & $\log _{10}\left(\tau_{m}\right)$ & $c_{m}$ \\
\hline Varying radius & 99.2 & 0.103 & 0.712 & 0.384 \\
Varying radius and resistivity & 95.6 & 0.103 & 0.716 & 0.387 \\
Varying radius and chargeability & 99.2 & 0.104 & 0.452 & 0.385 \\
Varying radius, resistivity and & 95.7 & 0.104 & 0.466 & 0.389 \\
chargeability & & & &
\end{tabular}

to characterize the upscaling of the Stern layer polarization and the membrane polarization.

\section{ACKNOWLEDGEMENTS}

We thank the reviewers and the Associate Editor for their useful comments. KT acknowledges the support of St. Petersburg State University (grant no. 3.37.134.2014).
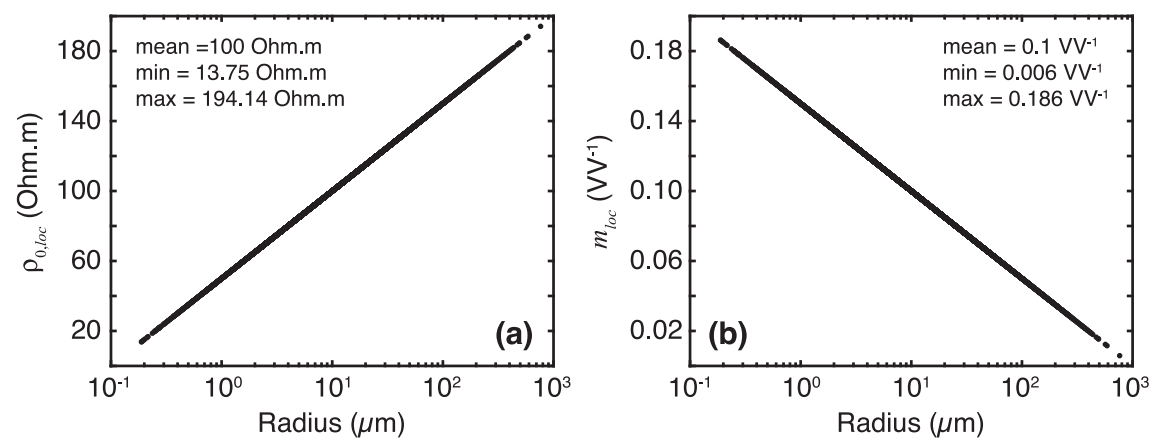

Figure 15. Evolution of the (a) local resistivity and (b) local chargeability versus tube radius chosen for a more realistic scenario of porous medium. 


\section{REFER E N CES}

Aarts, E.H.L. \& Van Laarhoven, P.J.M., 1985. Statistical cooling: a general approach to combinatorial optimization problems, Philips J. Res., 40, 193-226.

Abdel Aal, G.Z., Atekwana, E.A. \& Revil, A., 2014. Geophysical signatures of disseminated iron minerals: a proxy for understanding subsurface biophysicochemical processes, J. geophys. Res., 119, 1831-1849.

Attwa, M. \& Günther, T., 2013. Spectral induced polarization measurements for predicting the hydraulic conductivity in sandy aquifers, Hydrol. Earth Syst. Sci., 17, 4079-4094.

Bernabé, Y., 1998. Streaming potential in heterogeneous networks, J. geophys. Res., 103(B9), 20 827-20 841.

Bernabé, Y. \& Bruderer, C., 1998. Effect of the variance of pore size distribution on the transport properties in heterogeneous networks, J. geophys. Res., 103(B1), 513-525.

Bernabé, Y., Bruderer-Weng, C. \& Maineult, A., 2003. Permeability fluctuations in heterogeneous networks with different dimensionality and topology, J. geophys. Res., 108(B7), 2351, doi:10.1029/2002JB002326.

Bernabé, Y., Li., M. \& Maineult, A., 2010. Permeability and pore connectivity: a new model based on network simulations, J. geophys. Res., 115(B10), B10203, doi:10.1029/2010JB007444.

Bernabé, Y. \& Revil, A., 1995. Pore-scale heterogeneity, energy dissipation and the transport properties of rocks, Geophys. Res. Lett., 22(12), 15291552.

Bernabé, Y., Wang, Y., Qi, T. \& Li, M., 2016. Passive advection-dispersion in networks of tubes: effect of connectivity and relationship to permeability, J. geophys. Res., 121, 713-728.

Bernabé, Y., Zamora, M., Li, M., Maineult, A. \& Tang, Y.B., 2011. Pore connectivity, permeability, and electrical formation factor: a new model and comparison to experimental data, J. geophys. Res., 116(B11), B11204, doi:10.1029/2011JB008543.

Bohlen, T, 2002. Parallel 3-D viscoelastic finite difference seismic modelling, Comput. Geosci., 28(8), 887-889.

Bruderer, C. \& Bernabé, Y., 2001. Network modelling of dispersion: transition from Taylor dispersion in homogeneous networks to mechanical dispersion in very heterogeneous ones, Water Resour. Res., 37(4), 897908.

Bücker, M. \& Hördt, A., 2013a. Long and short narrow pore models for membrane polarization, Geophysics, 78(6), E299-E314.

Bücker, M. \& Hördt, A., 2013b. Analytical modelling of membrane polarization with explicit parameterization of pore radii and the electrical double layer, Geophys. J. Int., 194(2), 804-813.

Cole, K.S. \& Cole, R.H., 1941. Dispersion and absorption in dieletrics. I. Alternating current characteristics, J. Chem. Phys., 9, 341-351.

David, C., 1993. Geometry of flow paths for fluid transport in rocks, $J$. geophys. Res., 98(B7), 12 267-12 278.

David, C., Guéguen, Y. \& Pampoukis, G., 1990. Effective medium theory and network theory applied to the transport properties of rock, J. geophys. Res., 95(B5), 6993-7005.

Dias, C., 2000. Developments in a model to describe low-frequency electrical polarization of rocks, Geophysics, 65(2), 437-451.

Dukhin, S.S. \& Shilov, V.N., 2002. Non-equilibrium electrical surface phenomena and extended electrokinetic characterization of particles, in Interfacial Electrokinetics and Electrophoresis, Surfactant Science Series, Vol. 106, pp. 55-85, ed. Delgado, A.V., CRC.

Flores Orozco, A., Williams, K.H., Long, P.E., Hubbard, S.S. \& Kemna, A., 2011. Using complex resistivity imaging to infer biogeochemical processes associated with bioremediation of an uranium-contaminated aquifer, J. geophys. Res., 116(G3), G03001, doi:10.1029/2010JG001591.

Florsch, N., Camerlynck, C. \& Revil, A., 2010. Direct estimation of the distribution of relaxation times from induced-polarization spectra using a Fourier transform analysis, Near Surf. Geophys., 10(6), $517-531$.

Gallegos, D.P., Munn, K., Smith, D.M. \& Stermer, D.L., 1987. A NMR technique for the analysis of pore structure: application to materials with well-defined pore structure, J. Colloid Interface Sci., 119(1), $127-140$.
Holliger, K. \& Levander, A., 1992. A stochastic view of lower crustal fabric based on evidence from the Ivera zone, Geophys. Res. Lett., 19(11), 11531156.

Jackson, M., 2010. Multiphase electrokinetic coupling: insights into the impact of fluid and charge distribution at the pore scale from a bundle of capillary tubes model, J. geophys. Res., 115(B7), B07206, doi:10.1029/2009JB007092.

Johansson, S., Fiandaca, G. \& Dahlin, T., 2015. Influence of non-aqueous phase liquid configuration on induced polarization parameters: conceptual models applied to a time-domain field case study, J. appl. Geophys., 123, 295-309.

Kemna, A., Binley, A., Ramirez, A. \& Daily, W., 2000. Complex resistivity tomography for environmental applications, Chem. Eng. J., 77(1-2), $11-18$.

Kirchhoff, G., 1845. Ueber den Durchgang eines elektrischen Stromes durch eine Ebene, insbesondere durch eine kreisförmige, Ann. Phys., 140(4), 497-514.

Kirkpatrick, S., 1973. Percolation and conduction, Rev. Mod. Phys., 45, 574-588.

Koplik, J., 1981. On the effective medium theory of random linear networks, J. Phys. C: Solid State Phys., 14, 4821-4837.

Leroy, P., Revil, A., Kemna, A., Cosenza, P. \& Ghorbani, A., 2008. Complex conductivity of water-saturated packs of glass beads, J. Colloid Interface Sci., 321(1), 103-117.

Maineult, A., 2016. Estimation of the electrical potential distribution along metallic casing from surface self-potential profile, J. appl. Geophys., 129, 66-78.

Marshall, D.J. \& Madden, T.K., 1959. Induced polarization, a study of its causes, Geophysics, 24(1), 790-816.

Mewafy, F.M., Werkema, D.D., Atekwana, E.A., Slater, L.D., Abdel Aal, G.Z., Revil, A. \& Ntarlagiannis, D., 2013. Evidence that biometallic mineral precipitation enhances the complex conductivity response at a hydrocarbon contaminated site, J. appl. Geophys., 98, $113-123$.

Misra, S., Torres-Verdin, C., Revil, A., Rasmus, J. \& Homan, D., $2016 a$. Interfacial polarization of disseminated conductive minerals in absence of redox-active species. Part 1: mechanistic model and validation, Geophysics, 81(2), E139-E157.

Misra, S., Torres-Verdin, C., Revil, A., Rasmus, J. \& Homan, D., $2016 \mathrm{a}$. Interfacial polarization of disseminated conductive minerals in absence of redox-active species. Part 2: effective complex conductivity and dielectric permittivity, Geophysics, 81(2), E159-E176.

Nelder, J.A. \& Mead, R., 1965. A simplex method for function minimization, Comput. J., 7, 308-313.

Niu, Q. \& Revil, A., 2016. Connecting complex conductivity spectra to mercury porosimetry of sedimentary rocks, Geophysics, 81(1), E17-E32.

Ntarlagiannis, D., Robinson, J., Soupios, P. \& Slater, L., 2016. Field-scale electrical geophysics over an olive oil mill waste deposition site: evaluating the information content of resistivity versus induced polarization (IP) images for delineating the spatial extent of organic contamination, $J$. appl. Geophys., 135, 418-426.

Pelton, W.H., Ward, S.H., Hallof, P.G., Sill, W.R. \& Nelson, P.H., 1978. Mineral discrimination and removal of inductive coupling with multifrequency IP, Geophysics, 43(3), 588-609.

Revil, A., 2012. Spectral induced polarization of shaly sands: influence of the electrical double layer, Water Resour. Res., 48(2), W02517, doi:10.1029/2011WR011260.

Revil, A., 2013. Effective conductivity and permittivity of unsaturated porous materials in the frequency range $1 \mathrm{mHz}-1 \mathrm{GHz}$, Water Resour. Res., 49(1), 306-327.

Revil, A., Binley, A., Mejus, L. \& Kessouri, P., 2015. Predicting permeability from the characteristic relaxation time and intrinsic formation factor of complex conductivity spectra, Water Resour. Res., 51(8), 6672-6700.

Revil, A. \& Florsch, N. 2010. Determination of permeability from spectral induced polarization data in granular media, Geophys. J. Int., 181(3), $1480-1498$. 
Revil, A., Florsch, N. \& Camerlynck, C., 2014a. Spectral induced polarization porosimetry, Geophys. J. Int., 198(2), 1016-1033.

Revil, A., Kessouri, P. \& Torres-Verdín, C., 2014b. Electrical conductivity, induced polarization, and permeability of the Fontainebleau sandstone, Geophysics, 79(5), D301-D318.

Revil, A., Koch, K. \& Holliger, K., 2012. Is the grain size or characteristic pore size that controls the induced polarization relaxation time of clean sands and sandstones?, Water Resour. Res., 48(5), W05602, doi:10.1029/2011WR011561.

Ritter, H.L. \& Drake, L.C., 1945. Pore-size distribution in porous materials. Pressure porosimeter and determination of complete macropore-size distributions, Ind. Eng. Chem., 17(12), 782-786.

Rosen, L.A. \& Saville, D.A., 1991. Dielectrical spectroscopy of colloidal dispersions: comparisons between experiment and theory, Langmuir, 7(1), $36-42$.

Tarasov, A. \& Titov, K., 2013. On the use of the Cole-Cole equations in spectral induced polarization, Geophys. J. Int., 195(1), 352-356.
Titov, K., Komarov, V., Tarasov, V. \& Levitski, A., 2002. Theoretical and experimental study of time domain-induced polarization in water-saturated sands, J. appl. Geophys., 50, 417-433.

Turcotte, D.L., 1997. Fractal and Chaos in Geophysics, 2nd edn, Cambridge Univ. Press.

Veeken, P., Legeydo, P., Davidenko, Y., Kudryavceva, E., Ivanov, S. \& Chuvaev, A., 2009. Benefits of the induced polarization geoelectric method to hydrocarbon exploration, Geophysics, 74(2), B47-B59.

Weller, A. \& Slater, L., 2015. Induced polarization dependence on pore space geometry: empirical observations and mechanistic predictions, $J$. appl. Geophys., 123, 310-315.

Weller, A., Slater, L. \& Nordsiek, S., 2013. On the relationship between induced polarization and surface conductivity: implications for petrophysical interpretation of electrical measurements, Geophysics, 78(5), D315-D325.

Wong, J., 1979. An electrochemical model for the induced polarization phenomenon in disseminated sulfides ores, Geophysics, 44(7), 12451265 . 